\title{
Simulation of Route Optimization with load balancing Using AntNet System
}

\author{
Ashish Kumar Sharma ${ }^{1 *}$, Baijnath Kaushik ${ }^{2}$, A.K. Kohli ${ }^{3}$, Navdeep Kaur ${ }^{4}$ \\ ${ }^{I}$ Department of Computer Science, Krishna Engineering College, Ghaziabad, India \\ ${ }^{2}$ Department of Computer Science, Krishna Engineering College, Ghaziabad, India \\ ${ }^{3}$ Department of Electronics \& Communication, Thapar University, Patiala, India \\ ${ }^{4}$ Department of Information Technology, Chandigarh Engineering College, Chandigarh, India
}

\begin{abstract}
This paper is based on analysis of the performance of load balancing and route optimization in computerized networks. The complete system model shows the scenario of Packet distribution between nodes, and if congestion occurs due to traffic then Packet to be failed. The model used the AntNet system for simulate the network. The simulation runs on the ant's behavior for the load balancing of the network. The ants travel across the network between alternative chosen pairs of nodes; as they travel they deposit pheromones from their source node, collect the information of the route and the traffic congestion encountered on their journey. They select their path at each next node according the distribution of pheromones at each node. Packets between nodes are routed of the pheromone distributions at each next node. The performance of the network is proportional to packets which are failed. This model also shows the adaptivity of the system; the nodes are removed from the network, system finds the alternative chosen paths without system degradation and controls the performance of routing.
\end{abstract}

Keywords- AntNet Algorithm, Ant Colony Optimization (ACO), Routing, Load Balancing, Dynamic, Adaptive, Simulation, Communication Networks.

\section{Introduction}

In the communication network; congestion, delay and reliable communication play an important role to improve the performance and optimize the network and the network should be robust and adaptive. Optimization and performance are major issues in the industrial world as well as in the science world. So, these Issues occur in the communication network. Routing is the basic term of network because it handles the information as well as the control system performance. Routing is an important aspect of communication networks because it can greatly influence the overall network performance [3]; good routing can cause greater throughput or lower average delays and all other conditions would be same.

Load balancing [7] is essentially the construction of call-routing schemes which successfully distribute the changing load over the system and minimize lost calls. Of course it is possible to determine the shortest routes from every node to every other node of the network. In this way the average utilization of nodes will be minimized, but this is not necessarily the ideal way to avoid node congestion, idea should has to do with how the traffic on the network is distributed.

Routing is a distributed approach which is responsible for the routing tables, made on each node in the network, which give the information about incoming packets and which next link is used to continue the communication towards the destination node. Routing evolved some issues like user requirement, confliction between nodes and impact of different constraints of different technologies.

The AntNet is based on the ACO [14], which is a routing protocol for packed switched network invented by M. Dorigo and G. Di Caro [1]. ACO elaborates the nature and behavior of ants in a colony and could find the shortest path as well from the trail of the pheromone (chemical substance) deposited by the other ants. Through pheromone, ants could find out the shortest path using shortest path mechanism. After efforts of researchers on ants, a routing algorithm generated; which was realistic, and comparatively better than other algorithm. ACO $[6,7,8]$ improves the performance of the network and as well optimize with load balancing of the network. The problem of the network represented by the node graph, where artificial ants construct multiple paths from the source node to destination node and trace the path until optimal solution not found between two end node and it could take number of iteration in tracing of paths. Artificial ants collect the quantitative and qualitative information path cost and load of traffic in the network. These fundamentals are based on the ant colony system (ACS). ACS concept is used in AntNet [2]. An efficient routing algorithm will minimize the number of nodes that a packet will need to connect to in order to be completed thus; minimizing network load and increasing reliability. An implementation of AntNet based on Marco Dorigo and Thomas Stutzle has been designed. 


\section{Issues In Communication Network}

Routing is basic concept in communication network. It works to address the traffic between source node to destination node with determines the network performance. If routing has such importance then routing algorithms have the goals of directing data traffic from source to destination nodes in measure of network performance. The basic problem of routing is, to find the path of minimum cost between any two nodes that could be source node to destination node. Routing algorithms can be divided into three different categories; nonadaptive, adaptive, distributed. Non-adaptive routing algorithm is static routing, offline using local information, routing is not according to the traffic of network and the network topology. Adaptive routing $[3,4,5]$ algorithms are dynamic and centralized routing, to produce the optimal solution from the collected information through the entire network using global information. Distributed routing algorithms have mixture of both above algorithms that mean, uses mixture of offline local and global information. Routing algorithms strongly interact with congestion and admission control algorithms to determine the overall network performance.

The network performance depends on few constraints as like throughput (data delivered in unit time) and packet delay (sec). Throughput quantifies the quantity of service that the network has been able to offer in a certain amount of time, while packet delay defines the quality of service produced. The precise balance between throughput and average delay will be determined by the flow control algorithms operating concurrently with the routing algorithms. Along with these constraints; less congestion, high delivery rate, produce unexpected great performance, less overheads and less failure are also important measurements in the account.

In the network, problem associated define in the routing is as follows:

1. Find out the shortest path in the routing.

2. Selected shortest path might not be a minimum cost route.

3. Increase the through-put performance with less overhead.

4. Lacks of the least hop count.

5. Less effective congestion control, data flow is complex.

6. Load Balancing, Adaptive network

One of the issues with network routing (especially in very large networks such as the WAN, INTRANET etc.) is adaptability. Not only traffic can be unpredictably high, but the structure of a network can change as old nodes are removed and new nodes added. This perhaps, makes it almost impossible to find a combination of constant parameters to route a network optimally.

In this paper, we are concerned about the load balancing of the communication network with less failure of network and optimize the flow of traffic around the network using routing algorithm. According to the problems of network, optimize the network using AntNet $[9,10]$ and compare it a generic algorithm which is showing that AntNet performs better than another routing algorithm.

\section{AntNet: Adaptive Routing Algorithm}

The AntNet was proposed by M. Dorigo in 1997 and with modification in 1998 [1, 2, 5]. This paper is based on working of AntNet and solution implemented on it.

The operation of AntNet is based on two types of agents:

- Forward Ants who grab information about the status of network

- Backward Ants who grasp the information manipulate and update the routing tables of routers which is already situated on network.

The AntNet algorithm describes in steps:

1. On a regular time base, each source node sends a Forward Ant to a random destination.

2. In each node they pass, the elapsed time since the start is stored on an internal stack together with the identifier of the node. The Forward Ant selects the next hop in two way

- It draws between random nodes, where each random node has a probability to be selected.

- If the node is selected in previous way was already visited then it draws again the jumping node but now with the same probability for all neighbors.

3. If the selected node was already visited then a cycle is found. The Forward Ant pops from its stack all data of the cycle nodes, because the optimal path must not have any cycle.

4. When the Forward Ant reaches its final destination again the elapsed time since the start and the identifier of the node are stored on the stack of the ant. The Forward ant is converts into Backward Ant and transfers its stack. Backward Ant will return to source node with same path in opposite direction. 
5. The Backward Ants Use the information collected by the Forward Ants to update the random node routing table along the path. According to data carried in the stack increasing probabilities associated to the path used and decreases other paths probabilities.

6. When updation has been done and Backward Ant arrives on source node, stop working.

Due to algorithm, shows that Backward Ants have a higher priority than Forward Ants because Backward Ants has to have processes as fast as possible to make algorithm more adaptive comparison Forward Ants suffers from delays so that algorithm can fight with Congestion.

For Load balancing, the times that are saved onto the stack of the Forward Ants, are not computed as the difference between two timestamps. They are computed as the sum of two terms, one representing the delay due to link load, and one for the node load. When the load on a link becomes very high; the term representing the link load in increased to improve the load balancing [11] over multiple paths.

\section{Expermental Settings}

The bi-directional, un-weighted topological network of 30-node was chosen because this is a realistic interconnection structure of a possible switch-based network. It is also the same topology as was used in (Appleby \& Steward, 1994) [12], and is in fact the structure of the British Synchronous Digital Hierarchy (SDH) network.

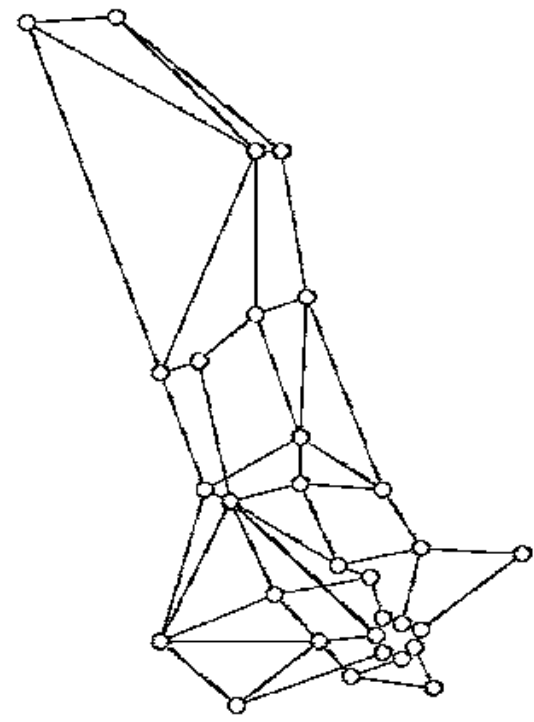

FIG- 1 The network topology is same as the SDH network of British Telecom and used in simulation.

The network is most naturally represented by an undirected graph. Each node in the graph corresponds to a switching station; the links between nodes correspond to communication channels. A given node will usually only be linked to a subset of other nodes, usually its geographical neighbors; links are bidirectional.

Every time step of the simulation proceeds as follows. First, packets that have expired are removed, releasing capacity at the nodes. Next, packets are sent by a traffic generator. These packets included a source node, a destination node and duration, measured in time steps. When a packet is sent, its route is determined by the current routing tables. The source and destination nodes are randomly chosen as a function of each node's probability of being an end node; this is both convenient and reasonable.

The simulator projected in Microsoft visual studio 2008 with the help of SQL server 2005 for the database of report. The complete simulation is based on the AntNet algorithm and following the criterion according the algorithm, whether it is related with parameters, design of network or working. In fact, simulator gives facility to examine the simulation through report of both AntNet On and Off mode and graph representation is plus point to compare the both result of modes. From running simulator, given tactics of simulation with respect of on and Off mode of AntNet [13]. The simulation having different tactics like speed (ticks/sec is a unit of near speed as system can run), packets, node capacity, concurrent packets and packet duration etc. Calculation will work on these fields of simulation.

\section{Results}

The experimental results are showing in the tables 1 to 2 and graphs 2 to 4 ; which are illustrate how the AntNet algorithm affects the routing of traffic. These tables showing the effectiveness of the algorithm against the system running without AntNet. Since it is possible to switch nodes on and off, a number of test 
comparisons will be done to show how AntNet can improve the routing of a network when paths are no longer valid and new routes have to be chosen.

When AntNet Simulator switched off that mean none of the pheromone values are read or written and work in generic algorithm.

The simulation ran numbers of times on few parameters. The Average best results obtained when values were changed with each and every simulation. The parameters values were as follows:-

i. Total packets- $10,50,100,150,200$

ii. $\quad$ Concurrent Packets- 4, 20, 40, 80, 120

iii. Node Capacity- 6, 30, 60, 100, 150

iv. Packet duration- 999 (same)

These parameters can run on different simulation speed as $1,5,10,50,100,1000$ ticks/sec which is almost similar to system speed.

Results shows with AntNet On and AntNet Off as follows:

- AntNet On gave better results than AntNet Off in Throughput (total packets send).

- AntNet On gave better results than AntNet Off in Current average packet.

- AntNet On gave better results than AntNet Off in Distance.

- AntNet On gave better results than AntNet Off in Delay.

\begin{tabular}{|l|l|l|}
\hline & AntNet ON & AntNet Off \\
\hline $\begin{array}{l}\text { Current Average } \\
\text { Packet }\end{array}$ & 6.49 & 6.08 \\
\hline Total Packets Send & 100 & 102 \\
\hline Distance & 185.69 & 267.35 \\
\hline Delay & .18569 & .26735 \\
\hline Failed Packets & 0 & 2 \\
\hline
\end{tabular}

Table 1 When Simulation Speed $=1000$ and Total Packets $=100$

Results are depends upon the simulation speed which is mention in above table and correspondingly mention different results which is elaborated that AntNet On is better than AntNet Off. Different results values is showing that whether, it's about total packet send over network, Distance and delay and failed packet but giving average best result over AntNet Off.

\begin{tabular}{|l|l|l|}
\hline & AntNet ON & AntNet Off \\
\hline $\begin{array}{l}\text { Current Average } \\
\text { Packet }\end{array}$ & 6.11 & 5.86 \\
\hline Total Packets Send & 100 & 101 \\
\hline Distance & 154.28 & 186.95 \\
\hline Delay & 1.5428 & 1.8695 \\
\hline Failed Packets & 0 & 1 \\
\hline
\end{tabular}

Table 2 When Simulation Speed $=100$ and Total Packets $=100$

Results is showing in table based on when simulation speed is 100 and total packets are same in previous table and still AntNet On providing average best result than AntNet Off. 


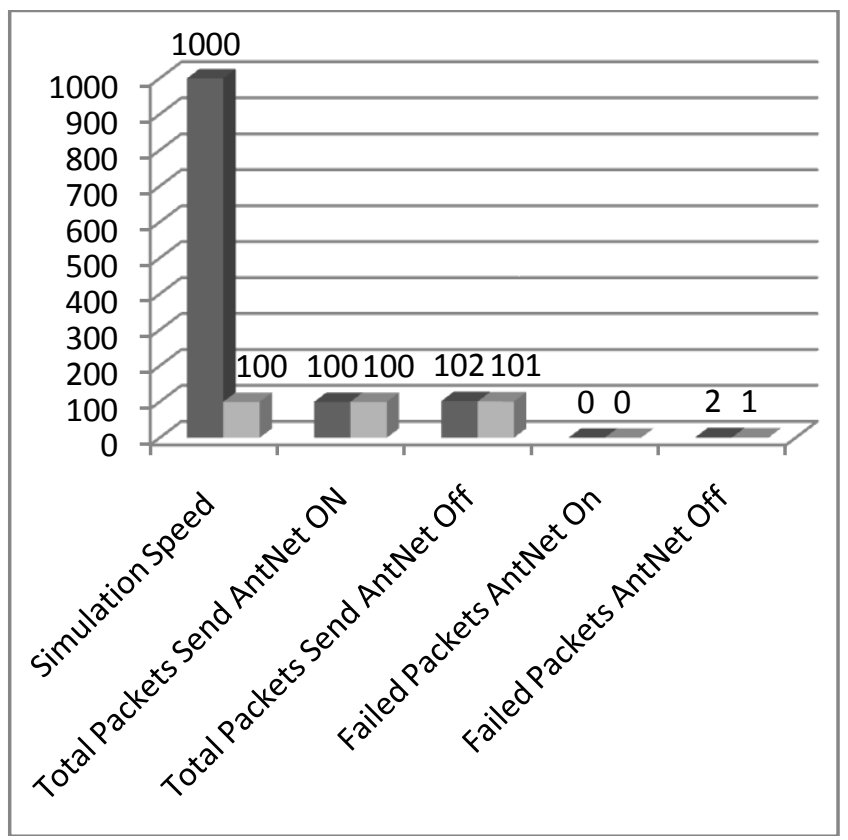

Fig.2 Simulation Speed vs. Total Packets Send and Failed Packets

In the Fig. 2, various results shown according simulation speed and differentiate how many packets send and how many packets failed.

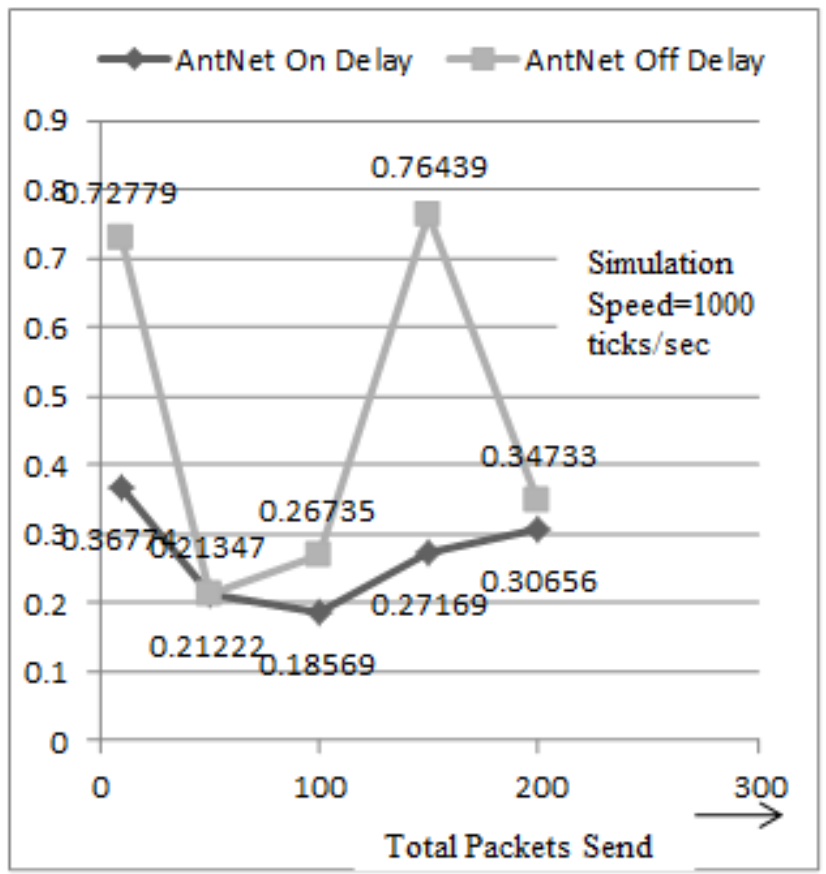

Fig. 3 Comparisons between Delay of AntNet On and Off.

In the Fig. 3, Packet delay is shown of both mode when simulation speed is 1000. Packet delays on nodes are less in AntNet On comparison in AntNet Off. 


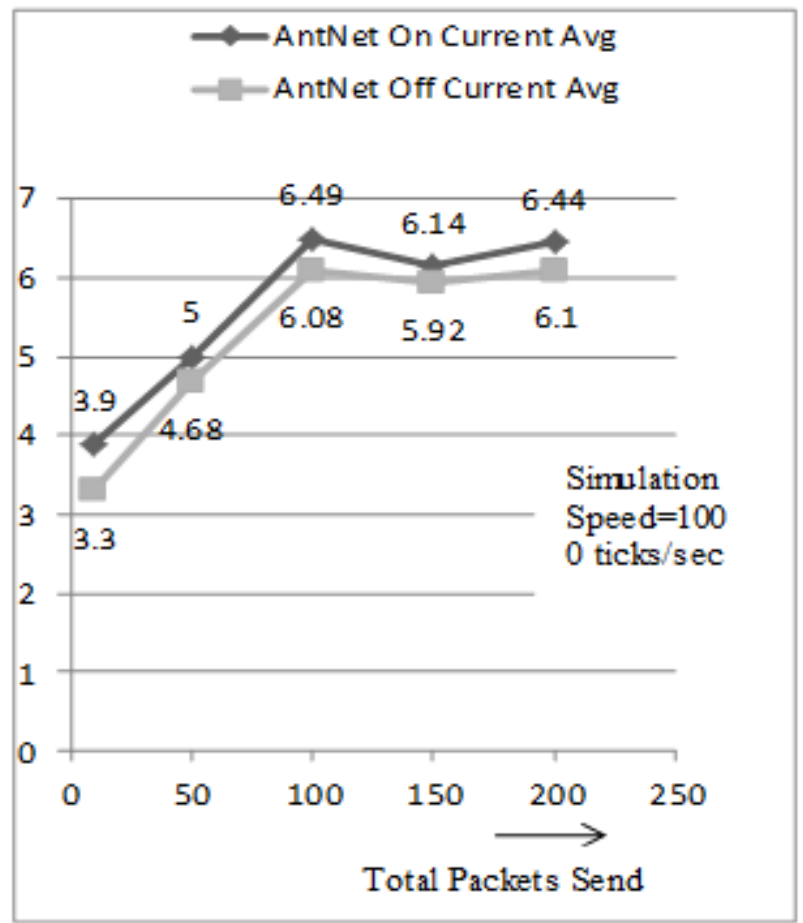

Fig.4 Comparisons between Current Average of AntNet On and Off.

In the fig. 4, Current Average packet sending is explaining that when we use AntNet On then its provides better results than AntNet Off.

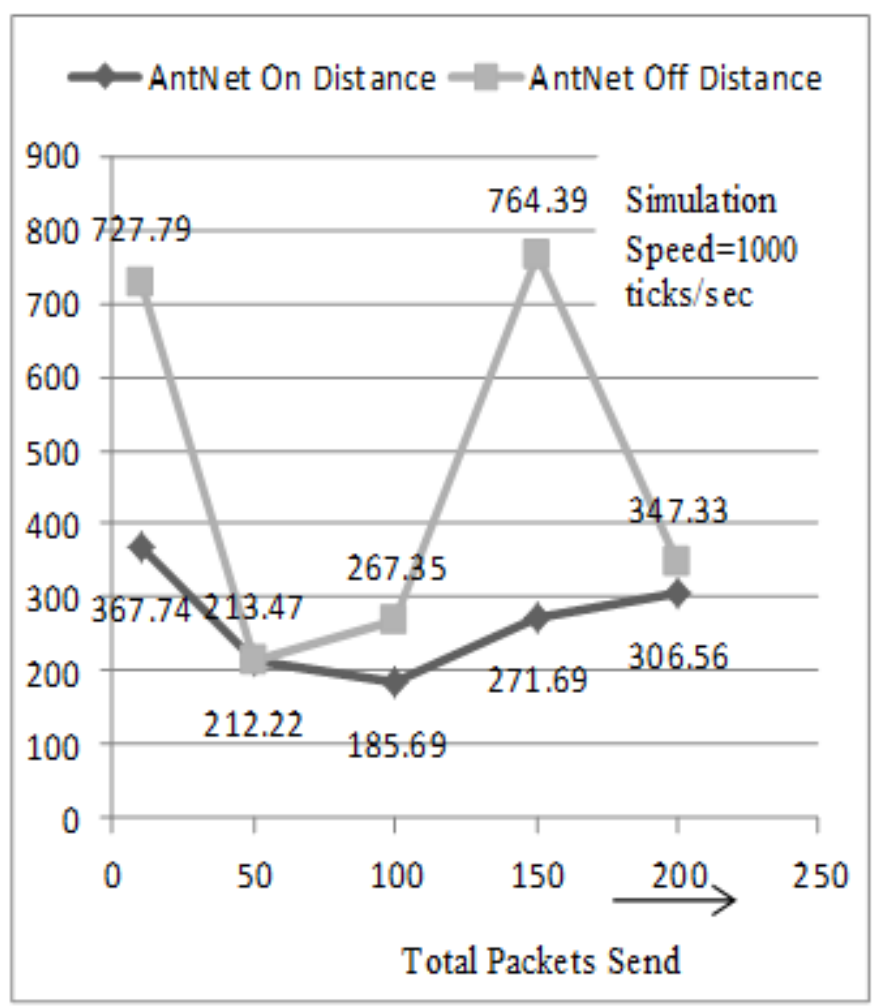

Fig. 5 Comparisons between Distance of AntNet On and Off.

In the Fig. 5, is same exactly like delay comparisons but here mention the distance between two nodes which is less in AntNet mode On. 


\section{Conclusion And Future Works}

The Result clearly shows that AntNet algorithm performs much better than Non AntNet algorithm and ant based load balancing fulfill technique. This was the basically a concept of packet switched telephony used in SDH British Telecom network. Further work could be that this simulation will work with real packet switched telecom system or VoIP. This simulation can be modified for more large number of nodes means it can be enhanced in future. In this scenario many concept remaining like evaporation, size of packets, and reaction of simulation after packet size etc. If we execute the simulation for a long time that means over a period of hours then processing of system reflects in a long way this concept is evaporation is remaining in and it can be resolute in future.

\section{References}

[1] G. Di Caro, M. Dorigo: AntNet: A Mobile Agents Approach to Adaptive Routing. Technical Report, IRIDIA, 1997.

[2] G. Di Caro, M. Dorigo: An adaptive multi-agent routing algorithm inspired by ants behavior. IRIDIA, Belgium, 1998.

[3] Benjamin Baran, Ruben Sosa: AntNet routing algorithm for data networks based on Mobile Agents. Inteligencia Artificial, 2001.

[4] G. Di Caro: Ant Colony Optimization and its Application to Adaptive Routing in Telecommunication Networks, IRIDIA, 2004

[5] G. Di Caro, M. Dorigo: AntNet: Distributed Stigmergetic Control for Communication Networks, Journal of Artificial Intelligence Research, 9, Vol. 9, PP. 317-365, IRIDIA, 1998.

[6] Benjamin Baran, Ruben Sosa: A New approach for AntNet Routing, Presented at the Proc. 9th Int. Conf. Computer Communications Networks, Las Vegas, NV, 2000.

[7] Ruud Schoonderwoerd, Owen Holland, Janet Bruten, Leon Rothkrantz: Ant-based load balancing in telecommunications networks, HP lab Bristol, U.K., Tech. Rep. HPL, 1997.

[8] E. Bonabeau, Florian Henaux, Sylvain Guerin, Dominique snyers, Pascale Kuntz, Guy Theraulaz: Routing in telecommunication networks with smart ant-like agents, Santa Fe Institute, 1999.

[9] V. Verstraete, M. Strobbe, E. Breusegem, Jan Coppens, Mario, Pickavet, and Piet Demeester: AntNet: ACO routing algorithm in practice, Ghent University, Belgium, 2002.

[10] M. Dorigo, G. Di Caro and L. M. Gambardella: Ant Algorithms for Discrete Optimization. Artificial Life, MIT Press, 1999

[11] S. Lipperts, Birgit Kreller: Mobile Agents in Telecommunications Networks - A Simulative Approach to Load Balancing, German Research (DFG), 1999

[12] S. Appleby, S. Stewards: Mobile Software agents for control in Telecommunication Networks, BT Technology Journal, Vol.12, no.2, 1994.

[13] Suchita Upadhyaya, Richa Setiya: Ant Colony Optimization: A modified Version, ICSRS, 2009.

[14] M. Dorigo, M. Birattari, T. Stutzle: Ant Colony Optimization IRIDIA, Technical Report, TR/IRIDIA/2006-023, September 2006. 